\title{
Mathematical model of power characteristics of the diagnostic fluorimeter
}

\author{
V.N. Grishanov ${ }^{1}$, V.S. Kulikov ${ }^{1}$, K.V. Cherepanov ${ }^{1}$

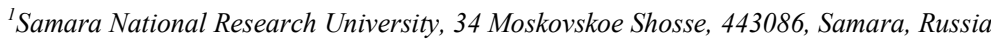

\begin{abstract}
A mathematical model for evaluation of power characteristics of the optical link of the fluorimeter is developed. The main objective of fluorimeter is measurement of intensity of fluorescent radiation of human skin in vivo. The model is realized in a packet of computer mathematics Mathcad and consists of the units modeling energetic characteristics of passive optical elements, radiators and photodetectors by analytic functions - laws of photometry. For creation of models elements reference, literary and experimental data on them are used. Basic purpose of model - operational quantitative comparing of constructive solutions for instrument by energetic criterion - photodiode`s output signal. The given obviously mathematical functions provide openness of model and accessibility for modification by the user.
\end{abstract}

Keywords: mathematical model; radiation stream; laser; light-emitting diode; photodiode; light filter; fluorimeter; photometry

\section{Introduction}

Human skin is the most available object of diagnostics in vivo. The skin integument is a peculiar accumulator of the products which reflecting the processes happening in an organism. Measurement of skin autofluorescence is demanded by physicians for an assessment of maintenance of the Advanced glycation end products (AGE). According to their contents complications in case of diabetes, coronary heart disease, operations on renal transplantation and a chronic hemodialysis are predicted [1 - 4]. Measurement of maintenance of AGE on the AFR level is used in dermatology for determination of a biological age of skin [5] and an assessment of activity of processes of biooxidation in fabrics [6]. Fluorescence of AGE is excited by radiation from the spectral range of 300 - 420 nanometers, and highlighting of radiation of fluorescence of AGE is watched in the spectral range of 420 - 600 nanometers.

In the West measurement of maintenance of AGE on the AFR level is accepted as one of required parameters for prediction risk of complications in cardiovascular system of the diabetic [7] and received the instrumental support in the form of family of the instruments AGE Reader of the DiagnOptics Technologies B.V company. [8], by means of which researches [1-3] are conducted. The instruments AGE Reader have the sizes of the netbook and are easy-to-work. The procedure of diagnostics doesn't exceed 5 minutes and consists that the patient puts forearm inside to an optical window of the instrument, and the operator clicks "Start-up". Results of diagnostics are displayed on a panel of the instrument and fixed in its memory. Fluorescence of AGE is excited by the mercury lamp, and is elastic dispersed by skin and fluorescent the radiations reaching an input end face of receiving optical fiber are transferred to them to a compact spectrometer. Diagnostic parameter in the instruments AGE Reader the integral criterion of the AFR [1,2] level appears

$$
A U=\frac{\int_{420}^{600} I_{f}(\lambda \lambda) d}{600-420} \times \frac{420-300}{\int_{300}^{420} I_{b s}(\lambda \lambda) d},
$$

where $I_{f}(\lambda)$ - a range of intensity of fluorescent radiation of skin in the range of lengths of waves $(420-600)$ nanometers; $I_{b s}(\lambda)$ - a range of intensity is elastic the radiation of excitation of fluorescence reflected by skin in the range of lengths of waves (300 - 420) nanometers. The experimental ranges of $I_{f}(\lambda)$ and $I_{b s}(\lambda)$ register the spectrometer which is an AGE Reader part.

Due to the lack of the AGE Reader equipment available to most medical institutions in Russia similar operations are carried out only at the research level [4-6, 9]. Researches are conducted on original universal spectrofluorometers [10-14] which operation assumes an involvement in it a highly qualified staff, there was no consensus also by diagnostic criterion [9].

In the Samara university with the assistance of authors of the real operation the diagnostic fluorimeter capable to solve the problem of measurement of AFR caused by AGE and the implementing integral diagnostic criterion (1) is created. From original, universal, research spectrofluorometers it shall differ in compactness, simplicity of construction and operation at the expense of optimized under the decision of the task set above by optical, electronic and algorithmic structures, and from instruments of the AGE Reader family - the budgetary element basis and easy replicability. The on-stage performance group managed to create two prototypes of the fluorimeter $[15,16]$ meeting the advanced criteria, first of which, single-channel, allowed to validate experimentally the made circuitry decisions by convincing demonstration of its ability to register very feeble radiation of AFR in the presence of destabilizing operational factors, and the second, being dual-channel, - as in laboratory, and in case of approbation in Regional clinical hospital of V. D. Seredavin showed ability to register age features of AFR and pathological processes at the patients having coronary heart disease.

As development is in a stage of optimization of designer decisions now, creation of simple mathematical models of the principal structural components of the diagnostic fluorometer is necessary for operational comparing of possible modifications of optical and optical-electronic elements and their relative positioning. 


\section{Object of simulation}

The optical circuit of the diagnostic fluorometer [16] is provided in fig. 1. Excitation of fluorescence of AGE which are contained in skin - a research object 5, is carried out by the radiation of a short-range ultra-violet or violet LED or the junction laser 1 which passes through the collimating optics 2 and the clearing light filter 3 . The range of radiation of excitation of AFR of 350 - 415 nanometers is caused by the AGE fluorescent properties [1-2,17], and in the specified range noticeable spectral non-uniformity efficiency of AFR excitation isn't marked that causes a designer level of freedom in a radiator choice.

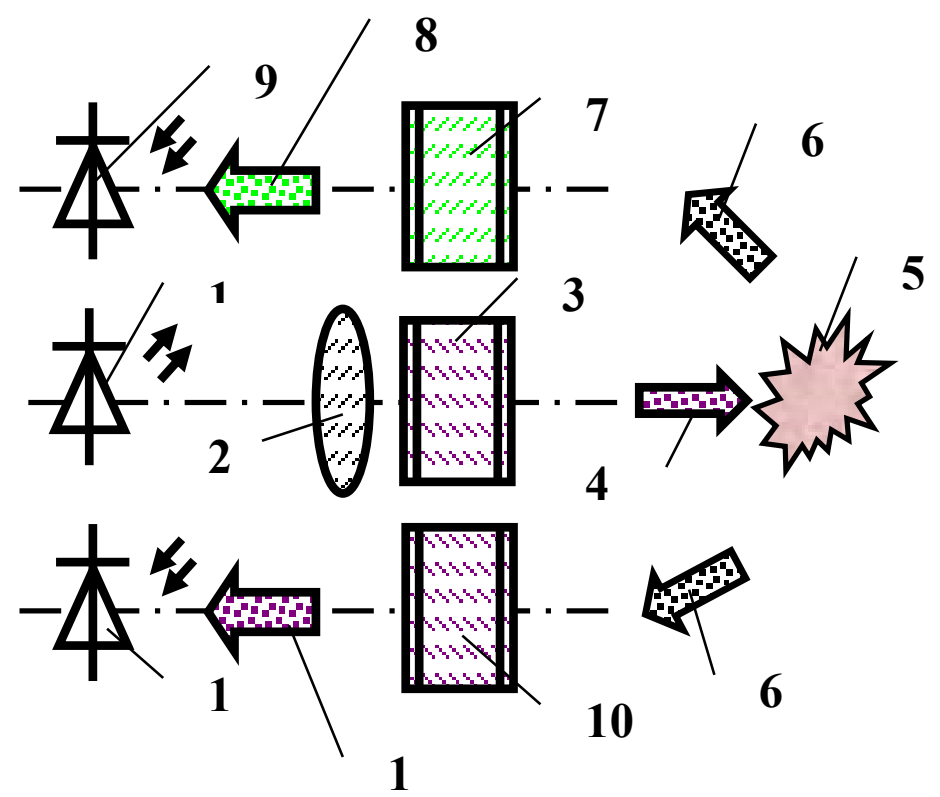

Fig. 1. Optical circuit of the diagnostic fluorometer: 1 - radiation source; 2 - the collimating optics; 3 - the clearing light filter; 4 - autofluorescence excitation radiant flux; 5 - the researched object; 6 - a compound it is elastic scattered and fluorescent radiations; 7 - the light filter which is cutting off fluorescence excitation radiation; 8 - flow of fluorescent radiation; 9 - photodiode of the channel of measurement of intensity of fluorescent radiation; 10 - the light filter which is cutting off fluorescent radiation; 11 - a flow it is elastic scattered radiation; 12 - the photodiode of the channel of measurement of intensity it is elastic scattered radiation.

Requirements of compactness and small energy consumption are narrowed by a choice to semiconductor sources of radiation. However it is impossible to recognize this restriction essential if for the solution of the main objective it is possible to pick up a LED or the junction laser of mass production, then on set of optical, operational and economic parameters it and will be, in most cases, the optimal designer solution [18].

Assignment of the clearing light filter 3 set in an exciting branch of the optical circuit consists in suppression of parasitic long-wave radiation which range is superimposed on a range of AFR [19]. Presence of additional long-wave radiation is characteristic of commercial UF and a blue range LEDs and its nature is described in operation [20]. It is caused by a radiant recombination in the upper layer of $\mathrm{p}-\mathrm{GaN}$ heterostructure of a LED. Intensity peak of parasitic long-wave radiation is in the ultra-violet and violet ranges next LEDs: LEUVS33G10TZ00, FYL-5013UVC, T5F36, EOLD-365-525, by the experimental estimates [19] made from 1'10-3 to 7,5'10-3 from intensity peak of the main radiation with a maximum on wavelength, inhering to an interval $(560 ; 580)$ nanometer. Elements $1-3$ form optical link of excitation of AFR. A part of the radiation dispersed by skin 6 through the light filter which is cutting off exciting radiation 7 falls on the photodiode 9 of the channel of measurement of intensity of AFR. The signal of the photodiode 9 is proportional to numerator of expression (1). Other part of the radiation dispersed by skin through the light filter which is cutting off fluorescent radiation 10 falls on the photodiode 12 of the channel of measurement of intensity is elastic scattered radiation. Its signal is proportional to a denominator of expression (1). The sketch of an optical system of the fluorometer is provided in fig. 2.

Object of simulation is also the optical system provided in fig. 1 and 2. Owing to geometrical symmetry of construction to channels for measurement intensity of elastic and fluorescent radiations the mathematical model of energetic characteristics shall describe dependence of the radiant flux falling on the photosensitive platform on one of photodiodes, and, therefore, and value of its output signal from spatial and energetic radiation parameters of excitation AFR set of such parameters of the photodiode as the sizes of its photosensitive site and indicatrix of sensitivity taking into account the dispersing research object properties. Optimized by means of model of energetic characteristics will be two key design parameters of an optical system: distance between optical axises of a excitation fluorescence source and the photodiode $\mathrm{b}$ and distance between a surface of the researched object and an input window of the photodiode.

The mathematical model of spectral characteristics shall provide an assessment photodiodes suitability on their spectral sensitivity; selection of type, materials and thickness of light filters for spectral dependences of their absorption coefficients or synthesis of a spectral characteristic of passage an interference light filter. Thus, important project and designer problems are also solved with its help. 

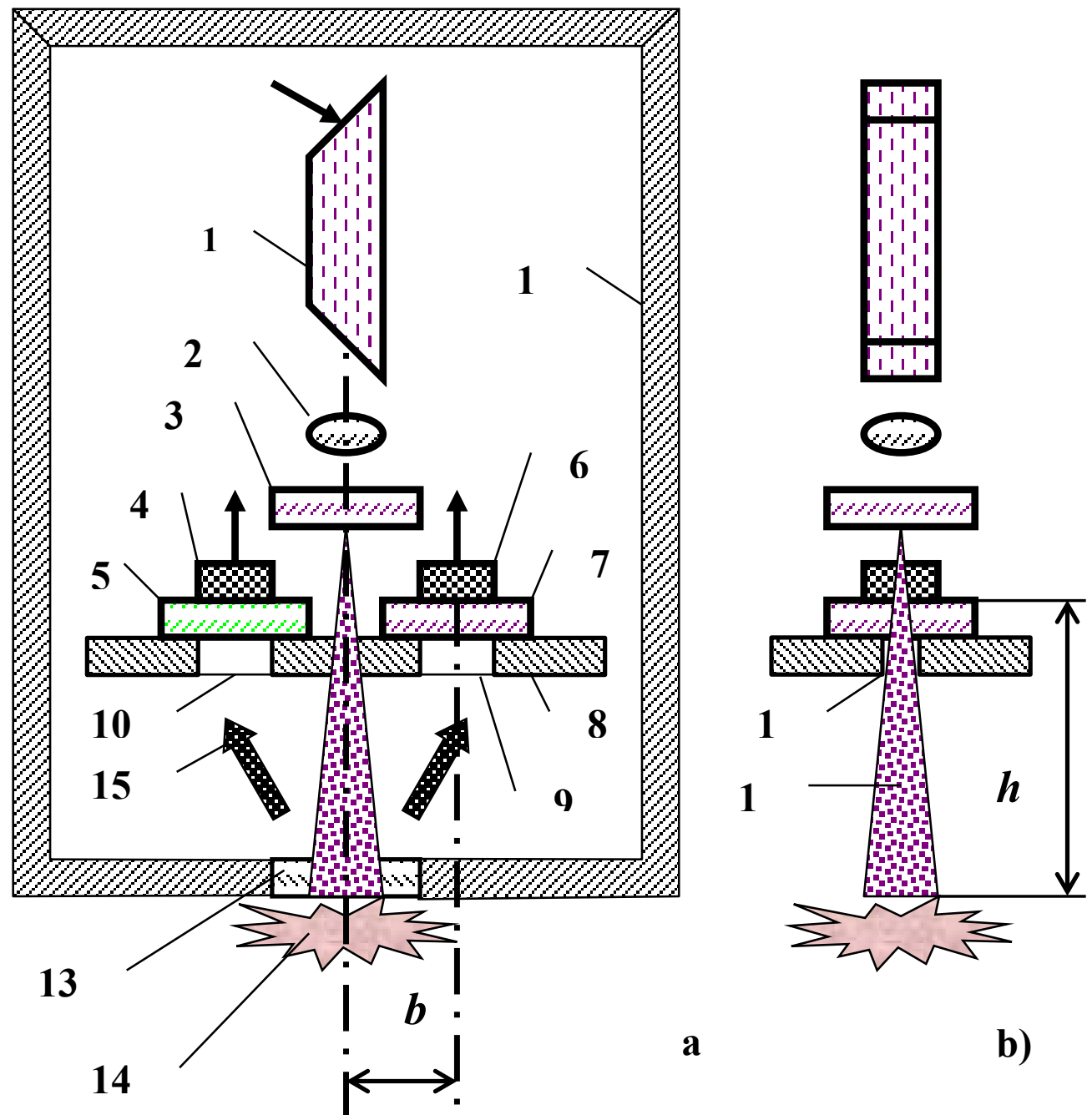

Fig. 2. Construction of an optical system of the diagnostic fluorometer: a) - frontal look; b) - side view; 1 - radiation source; 2 - the collimating optics; 3 - the clearing light filter; 4 - photodiode of the channel of measurement of intensity of fluorescent radiation; 5 - the light filter which is cutting off fluorescence excitation radiation; 6 - the photodiode of the channel of measurement of intensity it is elastic scattered radiation; 7 - the light filter which is cutting off fluorescent radiation; 8 - mounting plate; $9,10,11$ - holes in a mounting plate; 12 - lightproof casing; 13 - optical window; 14 - research object; 15 - a compound it is elastic scattered and fluorescent radiations; 16 - autofluorescence excitation radiant flux; $b$ - distance between optical axises of a source of excitation of fluorescence and the photodiode; $\mathrm{h}$ - distance between a surface of the researched object and an input window of the photodiode.

\section{Mathematical model of the energy characteristics}

Let us demonstrate the structure and operation of the energy characteristic model on the problem of optimizing the $b$ and $h$ design parameters. We propose using a laser module that includes the SLD3233VF semiconductor laser [21] and a built-in adjustable collimator as a radiation emitter in the design under development. Its peak emission wavelength is $405 \mathrm{~nm}$, and the peak emission power in a continuous mode is equal to $65 \mathrm{~mW}$. Due to the adjustable collimator, the emission power density in the examined skin area and the effective characteristic beam size can be varied within an order of magnitude. Therefore, the radiation emitter exciting SAF (skin autofluorescence) in the present paper is modeled by distributing the $E(x, y)$ power densityirradiance-over the object of investigation.

We expect to use as the emission detector the BPW21R silicon photodiode with an integrated light filter that shifts the peak of its spectral sensitivity to a wavelength of $560 \mathrm{~nm}$, which matches better with the AGE (advanced glycation endproducts) fluorescence spectrum. Since the photodiode manufacturers provide its $S(\alpha)$ sensitivity indicatrix [22], it is logical to consider a model of a point-contact photodetector the photodiode model to analyze the fluorimeter energy. The point-contact photodetector model features a defined direction of the sensitivity indicatrix axis, along which the sensitivity reaches its peak $S_{\max }=1$ value and the $\alpha$ angle, which determines the direction to the radiation emitter, is measured from it as well. The analysis of the angular dependence of $S(\alpha)$, given in graphical form in [22], showed that it is not differentiated from cosine:

$S(\alpha)=\cos \alpha$

The geometry of an optical system model is shown in Fig. 3. The object of investigation is considered flat and located in the $x O y$ plane. The skin test area has the shape of a square with $\alpha$ side. The origin of the reference system coincides with the geometric center of the examined area. The photodiode is located at a $P$ point belonging to the $y O z$ plane and is at a $b$ distance from the $z$ axis that is equal to the length of $P H$ or $O B$ segments and at a $h$ height from the $x O y$ plane equal to the length of $P B$ or $H O$ segments. The axis of the sensitivity indicatrix is directed vertically down along the $P B$ segment. 


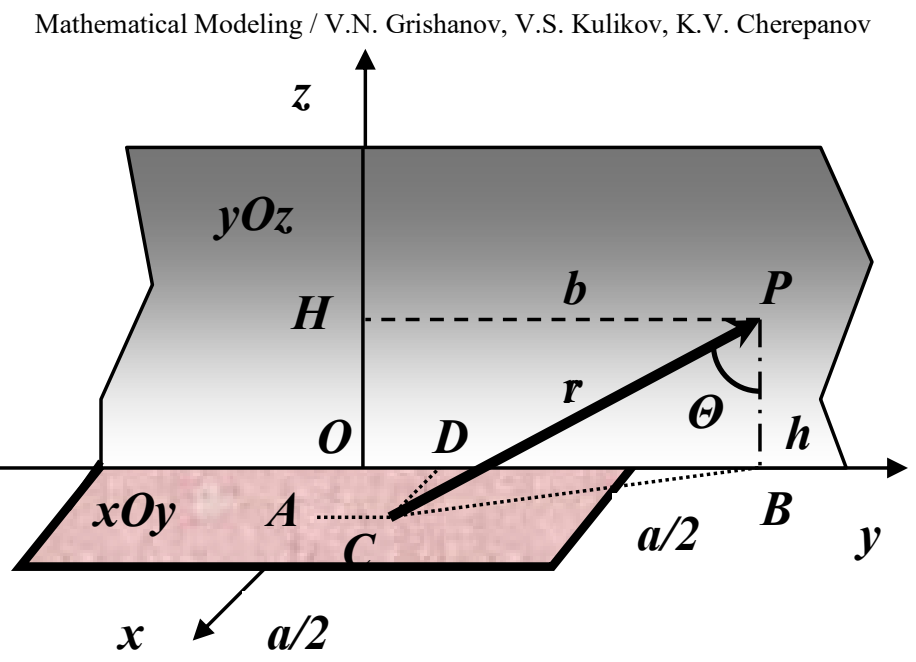

Fig. 3. Geometry of mathematical model of power characteristics.

The element of the $d x x d y$ scattering surface with the center at the $C$ point, the boundary point of the $C P$ segment that connects the surface element and the photodiode serves as an elementary radiation emitter for the photodiode. The $A$ and $D$ points are projections of the $C$ point onto the corresponding axes of reference. The $C B$ segment is a projection of the $C P$ segment onto the $x O y$ plane.

Since the surface of an examined object - skin - can be assumed to be Lambertian [23] in the first approximation with the $p$ reflectance at the angles of radiation incidence and radiation scattering up to $70^{\circ}$, its $L$ brightness will not depend on the scattering angle, and a brightness value of the surface element will be determined by its irradiance [24]:

$L(x, y)=\rho E(x, y) / \pi$,

Then the $d x x d y$ element will have normal radiation intensity:

$I_{0}(x, y)=L(x, y) d x d y=(\rho / \pi) E(x, y) d x d y$,

and the radiation intensity itself will vary according to the cosine law:

$I(x, y, \theta)=I_{0}(x, y) \cos \theta=(\rho / \pi) E(x, y) \cos \theta \cdot d x d y$,

where $\theta$ is the angle between the $C P$ segment and the normal to the $x O y$ plane. Denoting the length of the $C P$ segment by $r$, we obtain the irradiance created by the $d x x d y$ element at the location of the $P$ photodiode:

$E_{p}(x, y, \theta)=I(x, y, \theta) /[r(x, y)]^{2}$,

Denoting the irradiance transfer ratio into an electrical signal at the $k$ output of the photodiode, and taking into consideration its $S(\alpha)$ sensitivity indicatrix, we will have the following dependence of the $U$ output signal on the design parameters:

$U=(\rho k / \pi) \int_{-a / 2}^{a / 2} \int_{-a / 2}^{a / 2}\left\{E(x, y) /[r(x, y)]^{2}\right\} \cos \theta \cdot \cos \alpha \cdot d x d y$,

Since the axis of the sensitivity indicatrix is perpendicular to the $x O y$ plane, then we have $\alpha=\theta$ and from $\triangle C P B$ :

$\cos \alpha=P B / P C=h / r$.

From $\triangle C P B$ and $\triangle C D B$ we obtain the $r(x, y)$ dependence in explicit form:

$r(x, y)=\sqrt{C B^{2}+P B^{2}}=\sqrt{D B^{2}+C D^{2}+P B^{2}}=\sqrt{x^{2}+(b-y)^{2}+h^{2}}$,

which substituted in (8) and (7), results in the expression for the $U$ output signal of the photodiode:

$U=(\rho k / \pi) \int_{-a / 2}^{a / 2} \int_{-a / 2}^{a / 2}\left\{E(x, y) \cdot h^{2} /\left[x^{2}+(b-y)^{2}+h^{2}\right]^{2}\right\} d x d y$

that is convenient for calculations using the Mathcad software package.

\section{Optimization of the design parameters of a fluorimeter using a mathematical model}

Let us demonstrate the model optimization potential by solving the following problems: 1) using a wide or narrow beam to excite SAF, i.e. to select the window $a$ size; 2) the influence of the form of a spatial distribution of the $E(x, y)$ power density over an object per an output signal magnitude; 3 ) how sharply the output signal varies depending on a distance change between the $2 b$ photodiodes and 4 ) the $h$ height of their location above an investigated object. Since the optimization refers to a particular design shown in Fig. 2 with the selected type of BPW21R photodiode whose case diameter [22] is $9 \mathrm{~mm}$, we have $b \geq 4 \mathrm{~mm}$. It is not feasible to reduce the $h$ height to values less than $10 \mathrm{~mm}$ without complicating the optical system by using beam splitters, mirrors, etc. as well, i.e. we have $h \geq 10 \mathrm{~mm}$. Clinically tested devices $[8,16]$ diagnose a skin area with a characteristic size of 6 - $10 \mathrm{~mm}$. A diagnosed area of less than $1 \mathrm{~mm}$ in size can hardly be representative. Therefore, it is admissible to restrict the range of the $a$ parameter variation by the segment $[1 ; 10] \mathrm{mm}$. 
The optimization goal is to obtain a set of $a, b$ and $h$ geometric parameters with the design constraints discussed above that do not significantly reduce the $U$ output signal, all other things being equal. Then the value of the constant factor before the $(\rho k / \pi)$ integral (10) is taken equal to 1000 in order to obtain single-valued integers along the ordinate axis, the value of the $P_{u}$ emission power of the SAF exciting source is assumed equal to 1 , the $U$ output signal is measured in nominal units and the normalization condition is used:

$$
\int_{-a / 2}^{a / 2} \int_{-a / 2}^{a / 2} E(x, y) \cdot d x d y=1 \text {. }
$$

The uniform distribution of power density is the simplest one with simulation results easily verified physically: $E(x, y)=E_{0}=P_{u} / a^{2}=$ Const
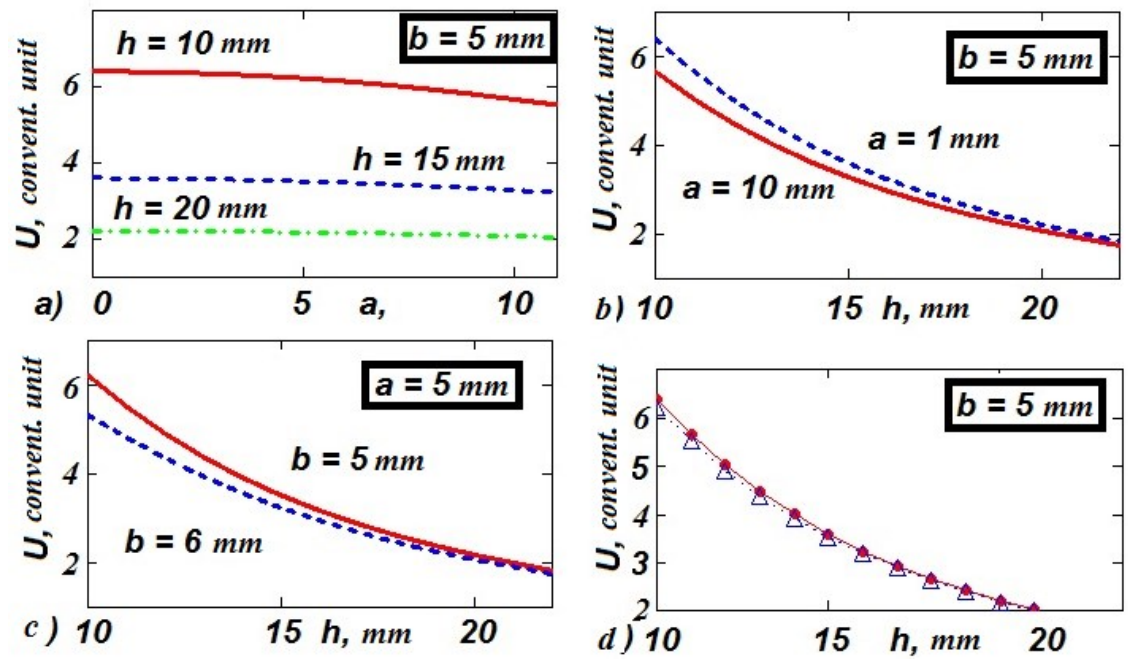

Fig. 4. Results of modeling (parameter in a frame has identical value for all curves of the schedule): a) - influence of the cross size of a bunch on the size of an output signal; b) - dependence of an output signal from photo diode arrangement height over a research object; c) - influence of distance of the photo diode from an optical axis of the probing bunch; d) - influence of the law of distribution of density of power of radiation of excitement of AFK on an object:: $\Delta \Delta \Delta-$

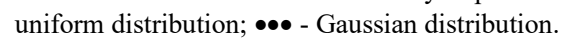

The simulation results are shown in Fig. 4. It follows from Fig. 4a that, in terms of the device energy, the formation of a small aperture beam does not provide any tangible advantage. The dependence on the height of the photodiode above the object of investigation appears to be more significant (Fig. 4b). It can be seen that this distance should be minimized; on the other hand, small $\sim 1 \mathrm{~mm}$ height variations caused, for example, by the need to replace a light filter with a light filter of a different thickness or errors in manufacturing optical element holders should not significantly affect the magnitude of an output signal. The last remark is related to the variation of the $b$ design parameter (Fig. 4c).

Fig. $4 \mathrm{~d}$ illustrates the effect of the law of the $E(x, y)$ power density distribution over the object. In addition to the uniform distribution, a Gaussian distribution is introduced into the model, as it is characteristic of laser radiation emitters:

$E(x, y)=M_{0} \exp \left[-2\left(x^{2}+y^{2}\right) / w^{2}\right]$

where $M_{0}$ is the power density on the Gaussian beam axis ; $w$ is the radius (the distribution parameter) of the beam. The normalization to the full power of the SAF exciting source is carried out with the help of the expression:

$M_{0}=2 P_{u} / \pi \cdot w^{2}$.

The comparison in Fig. 4d was carried out for the following values of the parameters: $a=5 \mathrm{~mm}$ and $\mathrm{w}=1.25 \mathrm{~mm}$. The choice of the value of a Gaussian beam radius was determined by the condition that the total radiation power of the emitter should almost completely fall on the object without masking it with an output window with a characteristic size of $5 \mathrm{~mm}$. The condition should be taken into consideration since it is known [25] that only $86.5 \%$ of the total power passes through the cross section of a Gaussian beam of $2 w$ diameter and to increase the total power to $99.99 \%$, the $4 w$ cross section diameter is required. As could be expected from physical considerations, according to the results of running the model with a uniform distribution of the power density of different cross sections (Figure 4a) the output signal is insensitive to the law of power density distribution, at least in the category of radially symmetric distributions. The $\left(U_{\text {gaussian }}-U_{\text {uniform }}\right) / U_{\text {gaussian }}$ relative difference of values does not exceed $2.5 \%$, which confirms the correctness of the mathematical apparatus used.

\section{Conclusion}

A mathematical model has been developed that enables to predict the energy characteristics of a device by automating a calculating component of designing according to the manufacturer's specifications, literature or experimental data on the parameters of optoelectronic system components of the designed diagnostic fluorimeter and the optical properties of a diagnosed object. 
Simulation showed that the most significant contribution to the device energy is made by the distance between the surface of a diagnosed object and the photosensitive pad of the photodetector. To obtain a maximum output signal of the photodetector, it is required to minimize the distance within the range of permissible design constraints. A small effect of the size of a diagnosed area on the output signal provides an additional degree of freedom for medical applications.

The model is implemented in the Mathcad software package to which mathematical models of the optical system components described above, the number of components and their parameters and expressions that the spectrum transfer over propagating radiation through an optical component obeys are introduced. The explicitly defined mathematical functions ensure the openness of the model and the feasibility of its modifying by a user.

\section{Acknowledgments}

This research was supported by the Ministry of Education and Science of the Russian Federation and results have been received within performance of the state task of the Ministry of Education and Science of the Russian Federation on the project 15.6567.2017/BCh (hands. V.P. Zakharov) - 15.6567.2017/8.9, and RFBR r_a (project 17-42-630907).

\section{References}

[1] Meerwaldt R, Graaff R, Oomen PHN et al. Simple non-invasive assessment of advanced glycation endproduct accumulation. Diabetologia 2004; 47: 1324-1330.

[2] Mulder D J, van Haelst PL, Graaff R et al. Skin autofluorescence is elevated in acute myocardial infarction and is associated with the one-year incidence of major adverse cardiac events. Netherlands Heart Journal 2009; 17(4): 162-168.

[3] Meerwaldt R, Hartog JWL, Graaff R et al. Skin Autofluorescence, a Measure of Cumulative Metabolic Stress and Advanced Glycation End Products, Predicts Mortality in Hemodialysis Patients. Journal of the American Society of Nephrology 2005; 16: 3687-3693.

[4] Golubev RV, Papayan GV, Glazunova AA, Korosteleva NYu, Petrishchev NN, Smirnov AV. Examination of skin autofl uorescence for the determination of glycation end-products in patients on chronic hemodialysis. Therapeutic Archive 2016; 88(6): 65-77.

[5] Papayan GV, Petrishchev NN, Krylova EV et al. Method of estimating the biological age of skin by means of a fluorescence multispectral video dermatoscope. Journal of Optical Technology 2010; 77(2): 60-67.

[6] Blyumin RB, Naumova EM, Khadartsev AA. The Technologies of Non-Contact Diagnostics. Journal of New Medical Technologies 2008. 15(4): 146149.

[7] Lutgers HL, Gerrits EG, Graaff R et al. Skin autofluorescence provides additional information to the UK Prospective Diabetes Study (UKPDS) risk score for the estimation of cardiovascular prognosis in type 2 diabetes mellitus. Diabetologia 2009; 52: 789-797.

[8] Page reader brochure: www.diagnoptics.com | www.age-reader.com.

[9] Dunaev AV, Dremin VV, Zherebtsov EA et al. Analysis individual variability of parameters of laser fluorescence diagnostics. Biotechnosphere 2013; 2(26): 39-47.

[10] Kang Uk, Papajan GV, Berezin VB et al. Spectrometer for fluorescence- reflective Biomedical Research. Journal of Optical Technology 2013; 80(1): 56-67. (in Russian)

[11] Papajan GV, Gurba VM, Kishalov AA et al. Fiber - reflective fluorescent spectrometer with multiwavelength excitation. Journal of Optical Technology 2014; 81(1): 38-43. (in Russian)

[12] Bulgakova NN, Smirnov VV, Fabelinsky VI et all. Laser spectral fluorescence colposcope: preclinical testing on experimental mice tumor. Biomedical 2013; 2: 108-122. (in Russian)

[13] Novikov IA, Grusha YO, Kiryushchenkova NP. Improving Efficacy of Fluorescent Diagnostics of Skin and Mucosal Tumors in Ocular Oncology. Annals of the Russian Academy of Medical Sciences 2012; 10: 62-69. (in Russian)

[14] Rogatkin DA, Sokolovski SG, Fedorova KA, Stewart NA, Sidorov VV, Rafailov EU. Basic principles of design and functioning of multifunctional laser diagnostic system for noninvasive medical spectrophotometry. SPIE Proc 2011; 7890: 1-7. DOI: 10.1117/12.874258.

[15] Kornilin DV, Grishanov VN. Portable fluorescence meter for medical applications. Proc. of SPIE 2016; 9887: 1-7. DOI: 10.1117/12.2227392.

[16] Kornilin DV, Grishanov VN, Zakharov VP, Burkov DS. Portable fluorescence meter with reference backscattering channel. Proc. SPIE 2016; 9961: 18. DOI: $10.1117 / 12.2237135$

[17] Koetsier M, Lutgers HL, Smit AJ, Links TP, de Vries R, Gans ROB, Rakhorst G, Graaff R. Skin autofluorescence for the risk assessment of chronic complications in diabetes: a broad excitation range is sufficient. Opt. Express 2009; 17: 509-519.

[18] Egorova OV, Schtejn GI. Comparison of fluorescence microscope lighting systems based on LEDs (LED) and a mercury lamp (HBO). Journal of Optical Technology 2011; 78(1): 99-101. (in Russian)

19. Grishanov VN, Kornilin DV, Kulikov VS. Adjustment of the emission spectra of the ultraviolet light-emitting diodes to excite the fluorescence of biological objects. Proc. of Actual problems of electronics and telecommunications: Russian Scientific and Technical Conference, Samara, 2015; 150 152.

[20] Jmerik VN, Mizerov AM, Shubina TV et al. Deep UV AlGaN quantum wells heterostructures grown by sub-monolayer digital molecular beam epitaxy with plasma-activated nitrogen. Physics and Technology Semiconductors 2008; 42(12): 1452-1457.

[21] Sld3233vf: www.alldatasheet.com/datasheet-pdf/pdf/228445/ETC2/SLD3233VF.html.

[22] Bpw21r: www.vishay.com.

[23] Barun VV et al. Light scattering by a rough surface of human skin. 1. The luminance factor of reflected light. Quantum Electronics 2013; 43(8): 768776.

[24] Yakushenkov YuG. Theory and calculation of optoelectronic devices. Moscow: Logos, 1999; 480 p.

[25] Klimkov YuM. Applied laser optics. Moscow: Mashinostroenie, 1985; 128 p. 